\title{
EVALUACIÓN DE LOS INDICADORES DE DETECCIÓN DE TUBERCULOSIS EN UNA REGIÓN CON ALTO RIESGO DE TRASMISIÓN EN PERÚ
}

\author{
Joel Roque-Henríquez ${ }^{1, a}$, Fresia Catacora-López ${ }^{2, b}$, Gladys Hilasaca-Yungas ${ }^{2, c}$, \\ Franco Romaní-Romaní,d
}

\begin{abstract}
RESUMEN
Se realizó una investigación operativa con el objetivo de evaluar los indicadores de detección de tuberculosis establecidos en la Norma Técnica para la Atención Integral de las Personas Afectadas por Tuberculosis. Se seleccionaron tres microrredes con muy alto riesgo de transmisión de tuberculosis de la región Tacna. Utilizando los libros de registro de sintomáticos respiratorios se analizaron los datos de 14595 sintomáticos respiratorios identificados (SRI), de los cuales se examinaron (SREx) 14486 (99,3\%), del total de SREx 1,5\% fueron BK (+). El promedio de baciloscopias directas por SRI fue de 2,0 y la intensidad de búsqueda (proporción de SRI entre las atenciones en > de 15 años) fue de 2,8\%. Los varones tuvieron mayor probabilidad de presentar, al menos, una baciloscopía positiva que las mujeres, OR 2,0 (IC 95\%: $1,5-2,6)$. Se concluye que el rendimiento de la baciloscopia diagnóstica en sintomáticos respiratorios es baja.
\end{abstract}

Palabras clave: Tuberculosis; Indicadores; Evaluación de programas (fuente: DeCS BIREME).

\section{EVALUATION OF TUBERCULOSIS DETECTION INDICATORS IN A REGION WITH HIGH RISK OF TRANSMISSION IN PERU}

\begin{abstract}
An operational study was conducted to evaluate the tuberculosis detection indicators established in the Technical Standard for Comprehensive Care of Persons Affected by Tuberculosis. Three networks of first level of care facilities with very high risk of tuberculosis transmission from the Tacna region were selected. Using the registry of respiratory symptomatic patients, we analyzed 14,595 respiratory symptomatic subjects (RSS), of which we examined (ExRSS) $14,486(99.3 \%)$. Of the total of ExRSS, 1.5\% were BK (+). The average direct smears per RSS was 2.0 and search intensity (ratio between RSS and number of health care visits in more than 15 years) was $2.8 \%$. Males were more likely to have at least one positive smear compared to women, OR 2.0 ( $95 \% \mathrm{Cl}: 1.5$ to 2.6$)$. It is concluded that the diagnostic performance of direct smears in respiratory symptomatic subjects is low.
\end{abstract}

Key words: Tuberculosis; Indicators; Program evaluation (source: MeSH NLM).

\section{INTRODUCCIÓN}

En el Perú, para el año 2012, la tuberculosis fue la segunda enfermedad infecciosa con mayor carga de enfermedad con 51597 años saludables perdidos (AVISA), solo después de las infecciones de vías respiratorias bajas que produjeron la pérdida de 275 173 AVISA (1). Según cifras oficiales del Ministerio de Salud, la incidencia acumulada (IA) de tuberculosis pulmonar frotis positivo (TBP-FP) ha disminuido de
161,1 por 100000 habitantes en 1993 hasta 58,6 por 100000 de habitantes en 2012. Existen nueve regiones que presentan incidencias acumuladas de TBP-FP por encima del promedio nacional, entre ellas la región Tacna que presenta una IA de 90,0 por 100000 habitantes (2), lo cual define un escenario epidemiológico de muy alto riesgo de transmisión de tuberculosis ${ }^{(3)}$.

Desde el año 2005 el número total de baciloscopías a nivel nacional sobrepasan los dos millones; en 2012

\footnotetext{
Universidad Peruana Cayetano Heredia. Lima, Perú.

Dirección Regional de Salud de Tacna. Tacna, Perú.

Oficina General de Investigación y Transferencia Tecnológica. Instituto Nacional de Salud. Lima, Perú.

Médico cirujano, magíster en Epidemiología Clínica; ${ }^{\mathrm{b}}$ licenciada en Enfermería, magíster en Enfermería en Salud del Niño y del Adolescente; ${ }^{\mathrm{c}}$ magíster en Enfermería, en Salud Familiar y Comunitaria; ${ }^{\mathrm{d}}$ médico cirujano.

* Los resultados provienen de la tesis para optar el grado de magíster en Epidemiología Clínica en la Universidad Peruana Cayetano Heredia realizada por Joel Roque Henríquez.

Recibido: : 23-01-15 Aprobado: 03-06-15
}

Citar como: Roque-Henríquez J, Catacora-López F, Hilasaca-Yngas G, Romaní-Romaní F. Evaluación de los indicadores de detección de tuberculosis en una región con alto riesgo de trasmisión en Perú. Rev Peru Med Exp Salud Publica. 2015;32(3):504-8. 
se realizaron 2549662 baciloscopías en 1400083 sintomáticos respiratorios examinados (2). Los costos estimados de la baciloscopías pueden variar entre países; en Brasil se ha estimado que el costo por baciloscopía es de 2,8 dólares americanos ${ }^{(4)}$, considerando dichos costos, el total de la inversión pública en baciloscopías sería considerable.

La proporción de baciloscopías positivas, entre el total de baciloscopias realizadas, ha sido evaluada en establecimientos de salud de un distrito de Lima Metropolitana encontrando un rendimiento del 2,0\% ${ }^{(5)}$, proporción similar a la encontrada en adultos tamizados para tuberculosis con menos de 14 días de tos (3,3\%); sin embargo, el rendimiento llega al $12,5 \%$ en sintomáticos respiratorios adecuadamente identificados ${ }^{(6)}$.

La adecuada aplicación de la definición de caso es un paso crítico en la mejora del rendimiento diagnóstico de la baciloscopía. La Estrategia Sanitaria Nacional de Prevención y Control de la Tuberculosis mide la intensidad de búsqueda de sintomáticos respiratorios, estableciendo por meta captar como sintomáticos respiratorios Identificados a cinco o más de cada 100 atenciones en pacientes mayores de 15 años (3). En este contexto, el objetivo del estudio es evaluar los indicadores operacionales de detección establecidas en la Norma Técnica de Salud para la Atención Integral de las Personas Afectadas por Tuberculosis, en tres microrredes de Tacna, con muy alto riesgo de transmisión.

\section{EL ESTUDIO}

\section{DISEÑO Y POBLACIÓN DE ESTUDIO}

La región Tacna, para el 2013, tuvo una población estimada de 333276 personas, dicha región cuenta con nueve microrredes de salud las que agrupan a 71 establecimientos del primer nivel de atención ${ }^{(7,8)}$. Para el 2013, Tacna presentó una incidencia acumulada de tuberculosis $\mathrm{BK}(+)$ de 87 por 100000 habitantes, y en los últimos diez años ha permanecido como una región de muy alto riesgo de transmisión de tuberculosis en el Perú. Se realizó una investigación operativa de exploración que incluyó al total de establecimientos de salud (EE.SS.) de tres microrredes: Cono Norte (que cuenta con siete establecimientos de salud); Metropolitana (siete establecimientos de salud), y Cono Sur (cinco establecimientos de salud) de la Red de Salud Tacna. Estas microrredes fueron seleccionadas debido a que en el 2013 tuvieron bajo su jurisdicción a los distritos de mayor incidencia acumulada de tuberculosis, y constituyen un escenario epidemiológico de "muy alto riesgo de transmisión" dentro de la región Tacna (Figura 1).

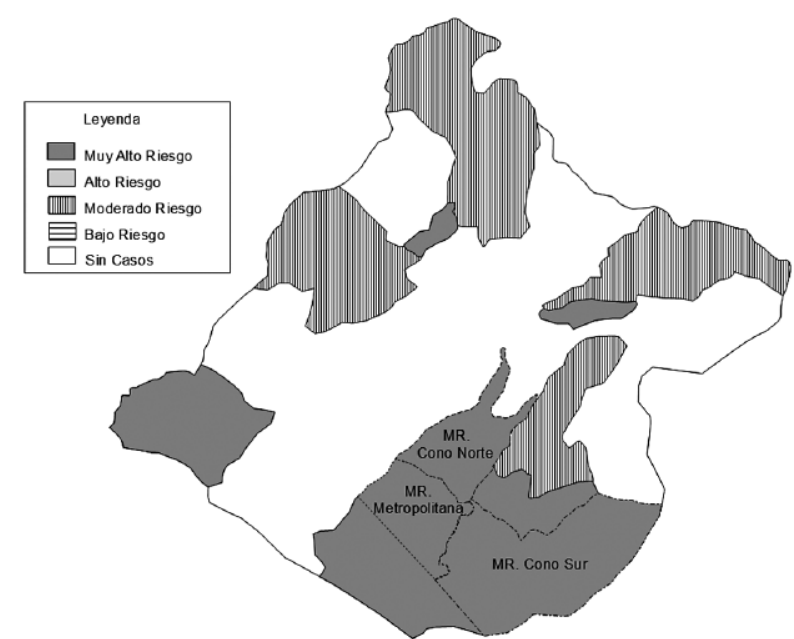

Figura 1. Mapa de riesgo de tuberculosis de la región Tacna, 2013

\section{DEFINICIONES OPERACIONALES}

En la Norma Técnica de Salud para la Atención Integral de las Personas Afectadas por Tuberculosis aprobada con Resolución Ministerial 715-2013/MINSA, se define como sintomático respiratorio (SR) a la persona que presenta tos y flema por 15 días o más; sintomático respiratorio identificado (SRI), al SR debidamente registrado en el libro de registros de sintomáticos respiratorios; sintomático respiratorio examinado (SREx), al SRI en el que se ha obtenido al menos un resultado de baciloscopía de diagnóstico; SREx Bk (+), al SREx con al menos un resultado de baciloscopía positiva; sintomático respiratorio esperado (SRE), a la proporción de SRI entre las atenciones en mayores de 15 años (mide la intensidad de búsqueda de SR con respecto a las atenciones en mayores de 15 años).

El rendimiento diagnóstico de la baciloscopia en SR ${ }^{6}$, es la proporción de SREx con baciloscopia positiva del total de SREx (evalúa la calidad de la detección SR).

\section{COLECCIÓN DE DATOS}

Se obtuvo copia del libro de registros de sintomáticos respiratorios así como el número de atenciones en mayores de 15 años correspondientes al año 2013 de los establecimientos de salud evaluados, previa coordinación y autorización de la Dirección Regional de Salud de Tacna (DIRESA Tacna). Se construyó una base de datos en Excel. Se incluyó en la base de datos a todos los SRI.

\section{ANÁLISIS ESTADÍSTICO}

Se calcularon los siguientes indicadores operacionales de detección: 
Proporción de SREx entre SRI, la cual tiene como meta el $100 \%$.

Proporción de SREx Bk (+) entre SREx. Si bien la Norma Técnica de Salud para la Atención Integral de las Personas Afectadas por Tuberculosis no consigna una meta, la meta de $10 \%$ figura en algunas investigaciones para lugares con alta incidencia dependientes de la detección pasiva de casos de tuberculosis, así como en la versión previa de la norma técnica nacional ${ }^{(3,6,9,10)}$.

Proporción de SRI entre las atenciones en mayores de 15 años, cuya meta es $\geq 5 \times 100$ atenciones.

Además, se calculó el promedio de baciloscopía directas por SRI. Y se exploró la correlación mediante el cálculo del coeficiente de Pearson entre intensidad de búsqueda y calidad de detección de SR como medidas ecológicas para cada uno de los EE.SS. de las tres microrredes. Se consideró como significativo un $p<0,05$. Los datos fueron analizados con el software STATA versión 13.

\section{CONSIDERACIONES ÉTICAS}

Se trabajó con una fuente de datos secundaria, para lo cual se obtuvo la autorización de la DIRESA Tacna. El equipo de investigación guardó la confidencialidad de los SR incluidos en la presente investigación. El estudio fue aprobado por el Comité Institucional de Ética para Humanos de la Universidad Peruana Cayetano Heredia (RCEI-14).

\section{HALLAZGOS}

Fueron 14595 SR debidamente registrados (SRI) en los libros de registro de sintomáticos respiratorios de las tres microrredes de salud, de los cuales en 14486 se obtuvo, al menos, un resultado de baciloscopía de diagnóstico (SREx). Del total de SRI, 8408 (57,6\%) fueron mujeres. La mediana de edad fue 34,8 años con intervalo intercuartil de 20 a 47 años y rango de edad entre 1 a 100 años. De los SRI, 12908 (88,4\%) fueron mayores de 15 años.

La Microrred de Salud Cono Norte representó el 44,7\% del total de SRI; la Microrred de Salud Metropolitana el $28,3 \%$, y la Microrred de Salud Cono Sur el 27,0\%.

Del total de SRI, en $14465(99,1 \%)$ se tuvieron los resultados de la primera muestra de esputo, en 13689 $(93,8 \%)$ los resultados de una segunda muestra y en 13 $668(93,6 \%)$ el resultado de ambas muestras de esputo. En 597 (4,1\%) se obtuvieron los resultados de más de dos muestras de esputo.

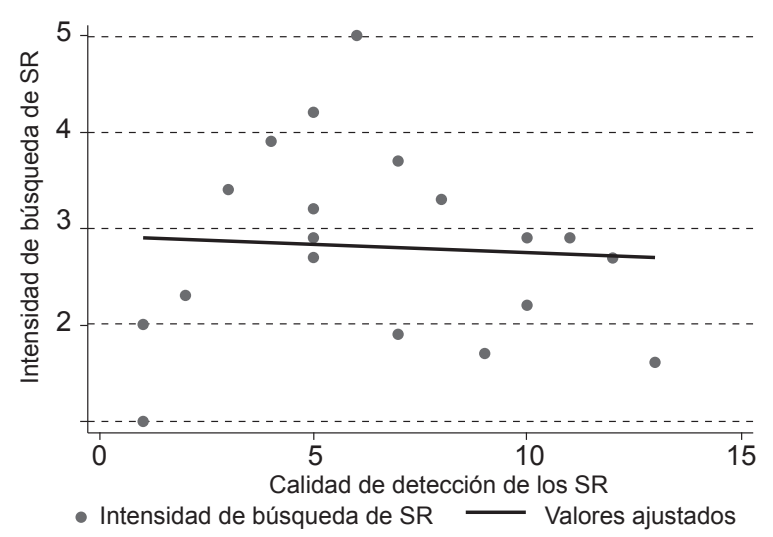

Figura 2. Correlación entre intensidad de búsqueda y calidad de detección de sintomáticos respiratorios en establecimientos de salud de tres microrredes de la Región Tacna.

No todos los SRI fueron examinados; la proporción total fue $99,3 \%$, con un rango de 93,2 a $100 \%$ según establecimientos de salud. El promedio de baciloscopias de diagnóstico por SRI fue de 2,0, con un rango de 1,8 a 2,1 según establecimientos de salud.

El porcentaje global de SREx Bk (+) entre el total de SREx (rendimiento diagnóstico de la baciloscopía en SR) fue de 1,5\%; ningún establecimiento de salud alcanzó la meta del $10 \%$, en dos de ellos este resultado fue de $0 \%$; siendo el mayor rendimiento de un E.S. de $4,0 \%$. Los EE.SS. de la Microrred de Salud Cono Norte alcanzaron un rendimiento diagnóstico del 1,4\%, los de la Microrred Metropolitana y Cono Sur tuvieron 1,6\% respectivamente.

El mayor rendimiento diagnóstico se dio en la etapa de vida joven (2,3\%). En aquellos con dos o más muestras de esputo el rendimiento fue de 1,6\%. Noventa $(1,1 \%)$ de las mujeres SREx presentó, al menos, una baciloscopía positiva, mientras que en los varones la cantidad fue de $129(2,1 \%)$. Los varones tuvieron mayor probabilidad de presentar, al menos, una baciloscopía positiva que las mujeres, siendo el OR 2,0 (IC 95\%: 1,5-2,6). En prácticamente todas las etapas de vida la proporción de positivos fue mayor en varones (Tabla 1).

La proporción de SRI mayores de 15 años entre las atenciones en mayores de 15 años (SRE) fue de 2,8\%. Dicha proporción varió por establecimientos de salud entre 1,0 a $5,0 \%$; solo un establecimientos de salud de los 19 estudiados logró la meta del 5\%. La Microrred Cono Norte tuvo una intensidad de búsqueda del 3,0\%, la Microrred Metropolitana del 2,5\% y la Microrred Cono Sur del 2,9\%. En la Figura 2 se aprecia una discreta relación inversa de los indicadores de rendimiento diagnóstico de la baciloscopía en SR con el de intensidad 
Tabla 1. Rendimiento diagnóstico de la baciloscopía en SR según etapa de vida

\begin{tabular}{lccc}
\hline $\begin{array}{l}\text { Etapa de vida } \\
\text { (rango de edad) }\end{array}$ & Varones & Mujeres & Total \\
\cline { 2 - 3 } Niño (0-11) & SREx Bk (+)/SREx (\%) & SREx Bk (+)/SREx (\%) & SREx Bk (+)/SREx (\%) \\
\hline Adolescente (12-17) & $0 / 375(0,0)$ & $0 / 403(0,0)$ & $0 / 778(0,0)$ \\
Joven (18-29) & $22 / 889(2,5)$ & $7 / 857(0,8)$ & $29 / 1746(1,7)$ \\
Adulto (30-59) & $61 / 2035(3,0)$ & $43 / 2520(1,7)$ & $104 / 4555(2,3)$ \\
Adulto mayor $(\geq 60)$ & $35 / 1942(1,8)$ & $32 / 3407(0,9)$ & $67 / 5349(1,3)$ \\
Total & $11 / 887(1,2)$ & $8 / 1171(0,7)$ & $19 / 2058(0,9)$ \\
\hline
\end{tabular}

SREx BK (+): sintomático respiratorio examinado con baciloscopía positiva SREx: sintomático respiratorio examinado

de búsqueda de SR con respecto a las atenciones en mayores de 15 años (coeficiente de correlación de Pearson $=-0,1$; Valor $p=0,8)$.

\section{DISCUSIÓN}

El principal hallazgo es el bajo rendimiento que tuvo la baciloscopía directa para la captación de casos de tuberculosis pulmonar. En tres microrredes de la región Tacna con muy alto riesgo de transmisión de tuberculosis, la baciloscopía resultó positiva en 15 de cada 1000 SREx.

En los últimos años, Perú mediante el programa presupuestal TBC-VIH/SIDA, ha invertido mayor cantidad de presupuesto en el producto despistaje de tuberculosis en sintomáticos respiratorios. En dicho producto, Tacna en los años 2011, 2012 y 2013, ha invertido aproximadamente 94500,137000 y 314800 USD respectivamente, parte de este gasto se encuentra destinado a la realización de la baciloscopia directa. Considerando un costo de 0,50 USD por baciloscopia directa en dos muestras de esputo en países de bajos recursos, y que en los 19 establecimientos de salud analizados se realizaron en total 29271 baciloscopías (11), se estima que en las microrredes estudiadas en un año se gastó 14 635,5 USD (esta estimación no considera costos indirectos).

El problema del bajo rendimiento de la baciloscopía en Perú ha sido reportado en estudios previos; un estudio realizado en cuatro establecimientos de salud del distrito de Santa Anita (Lima), en el 2012, encontró un rendimiento del 2,4\% (5), otro estudio realizado en seis establecimientos de salud del distrito de San Juan de Lurigancho entre el 2003 y 2005 encontró un rendimiento del 7,3\% ${ }^{(6)}$. Rendimientos diagnósticos superiores han sido reportados en estudios realizados en otros países, en Vietnam (10,3\%) ${ }^{(9)}$, Etiopía $(10,9 \%)^{(10)}$ y Tanzania $(18,9 \%)^{(12)}$

Se encontraron diferencias en el rendimiento de la baciloscopía según sexo, los varones tuvieron dos veces la probabilidad de presentar, al menos, una baciloscopía positiva comparado con las mujeres, dicho hallazgo es consistente con otros estudios ${ }^{(5,9)}$. Las razones de estas diferencias no están bien comprendidas y escapan a los objetivos de esta investigación; sin embargo, estas no se deberían a diferencias de acceso a los servicios de salud (en el presente estudio el mayor porcentaje de SRI en 18 de los 19 establecimientos de salud fueron mujeres) o diferencias en el número de muestras, en nuestro estudio el promedio de número de muestras de esputo por cada SRI mujer y varón fue de 2,0. Diferencias en la calidad de la muestra de esputo es una explicación poco probable según Huong et al. ${ }^{(9)}$.

Existe un adecuado cumplimiento de otros indicadores como el de capacidad del personal de salud para realizar la baciloscopía entre los SRI (99,3 de una meta de $100 \%$ ) y promedio de baciloscopias por SRI (dos de una meta de dos), lo cual es consistente con un estudio previo usando la misma metodología ${ }^{(5)}$.

No se encontró correlación entre la intensidad de búsqueda y la calidad de detección de sintomáticos respiratorios, consideramos necesario la realización de estudios que determinen con mayor precisión esta asociación. Por otro lado, es necesaria la evaluación de la utilidad del indicador intensidad de búsqueda, publicaciones internacionales no evalúan este indicador ${ }^{(6,9,10,12)}$ y es necesario generar evidencia que respalde su uso.

En la presente investigación operativa se midieron todos los indicadores operacionales de detección a diferencia de los estudios previos realizados en Perú ${ }^{(5,6)}$. Además, abarca un mayor número de establecimientos de salud, en total 19 establecimientos de salud correspondientes a las tres microrredes con mayor riesgo de tuberculosis de la región Tacna.

Entre las limitaciones del presente estudio se encuentra la fuente de información utilizada, el libro de registros de sintomáticos respiratorios puede contener errores como parte de su llenado de rutina; por otro lado, no se estudiaron las razones para el bajo rendimiento de la baciloscopía, como la calidad de las muestras y de la ejecución de 
las pruebas de laboratorio. Finalmente, se recomienda diseñar un estudio prospectivo para la región Tacna que determine los factores asociados al bajo rendimiento de la baciloscopía en esputo, así como evaluar las metas para los indicadores operacionales de detección de tuberculosis establecidas en la Norma Técnica.

Agradecimientos: al Dr. Herminio Hernández Díaz por su valiosa asesoría a la presente investigación. A la DIRESA Tacna por las facilidades dadas para la realización de este estudio.
Contribuciones de autoría: JRH y FRR participaron en la concepción, análisis e interpretación de datos, redacción del artículo, revisión crítica del artículo y aprobación de versión final. FCL y GHY participaron en la concepción, asesoría técnica y recolección de datos.

Fuentes de financiamiento: autofinanciado.

Conflictos de interés:los autores declaran no tener conflictos de interés.

\section{REFERENCIAS BIBLIOGRÁFICAS}

1. Valdez W, Miranda J; Perú, Ministerio de Salud. Carga de enfermedad en el Perú: Estimación de los años de vida saludables perdidos 2012. Lima: MINSA-DGE; 2014.

2. Perú, Ministerio de Salud. Sala Situacional Tuberculosis 2012. Lima: Ministerio de Salud; 2013.

3. Perú, Ministerio de Salud. Norma técnica de salud para la atención integral de las personas afectadas por tuberculosis. NTS No 104 - MINSA/ DGSP-V.01 2013. Lima: MINSA; 2013.

4. TB Diagnostics Market Analysis Consortium. Market assessment of tuberculosis diagnostics in Brazil in 2012. PLoS One. 2014 Aug 6;9(8):e104105. doi: 10.1371/journal. pone. 010410 .

5. Roque-Henríquez J, Romaní-Romaní F, Eunbee-Cho C, Contreras-Mendoza M, Salinas-Castro W. Rendimiento diagnóstico de la baciloscopía en sintomáticos respiratorios usuarios de establecimientos de salud del primer nivel en un distrito de Lima Metropolitana. Rev Peru Epidemiol. 2013;17(2):1-6.

6. Otero L, Ugaz R, Dieltiens G, González E, Verdonck K, Seas C, et al. Duration of cough, TB suspects' characteristics and service factors determine the yield of smear microscopy. Trop Med Int Health. 2010;15(12):1475-80. doi: 10.1111/j.1365-3156.2010.02645.x.

7. Perú, Ministerio de Salud. Análisis de Situación de Salud del Perú. Lima: MINSA-DGE; 2013.

8. Instituto Nacional de Estadística e Informática. Perú en cifras. Lima: INEI; 2014.

9. Huong NT, Duong BD, Linh NN, Van LN, Co NV, Broekmans JF, et al. Evaluation of sputum smear microscopy in the National Tuberculosis Control Programme in the north of Vietnam. Int J Tuberc Lung Dis. 2006;10(3):277-82.

10. Ali H, Zeynudin A, Mekonnen A, Abera S, Ali S. Smear Posetive
Pulmonary Tuberculosis (PTB) Prevalence Amongst Patients at Agaro Teaching Health Center, South West Ethiopia. Ethiop J Health Sci. 2012;22(1):71-6.

11. Molicotti P, Bua A, Zanetti S. Costeffectiveness in the diagnosis of tuberculosis: choices in developing countries. J Infect Dev Ctries. 2014;8(1):24-38. doi: 10.3855/ jidc.3295.

12. Ipuge YA, Rieder HL, Enarson DA. The yield of acid-fast bacilli from serial smears in routine microscopy laboratories in rural Tanzania. Trans $\mathrm{R}$ Soc Trop Med Hyg. 1996;90(3):258 61.

Correspondencia: Joel Roque Henríquez Dirección: Cápac Yupanqui 1400, Jesús María. Lima, Perú

Teléfono: (511) 940430215

Correo electrónico:jroque@ins.gob.pe

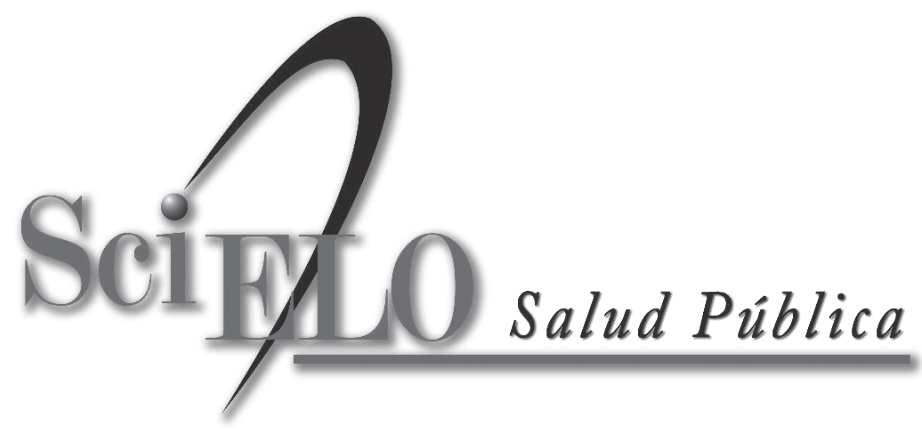

